CS-only extinction trials following the peak acquisition conditioned GSR extinguished in an average of 42 trials, while Schramm \& Kimmel (1970) obtained an average of 44 trials under the same conditions. If the 16 unpaired CSs and UCSs of the present paired-unpaired controls are assumed to be the approximate equiralent of 16 extinction trials, then these Ss reached the nonresponse criterion in a total of 18.6 trials (i.e.. 16 plus 2.6), still substantially less than the values obtained in the standard CS-only extinction condition.

(Received for publication November 5. 1973.)

\title{
The effect of differential overtraining of the positive and negative stimulus on the aversiveness of the negative stimulus
}

\author{
ISAAC BEHAR* \\ United States Army Medical Research Laboratory, Fort Knox, Kentucky 40121
}

\begin{abstract}
Six discrimination-sophisticated monkeys were trained on 18 problems, each of which contained intermixed trials on two discriminations. In six problems, the ratio of trials of the two discriminations was 4 to 1 . Following training, a single test trial was given, which consisted of choice between the more trained (MT) and less trained (LT) negative stimuli. No consistent preference for either stimulus was demonstrated. In the remaining problems, either the negative or positive stimulus of one discrimination was overtrained. In the former condition, on test trials, the LT negative stimuli were preferred, while in the latter, the MT negative stimuli were preferred. These results could be accounted for by the relative frequency during training of nonrewarded responses to the MT and LT negative stimuli.
\end{abstract}

The nonreinforcement of a response to the negative stimulus in a two-choice simultaneous discrimination appears, in naive animals, not to be aversive, at least in the sense that the probability of response to the stimulus has not been reduced (Behar, 1961). In the study noted, naive monkeys responded about equally as often to a stimulus that was associated with no reward on half of the trials and a double reward on the remaining trials as they did to a stimulus that was associated on all trials with a single reward. Discrimination learning-set sophisticated monkeys, on the other hand, strongly avoided the stimulus associated with partial nonreward.

*This experiment developed out of a discussion with Stanton Wharburton III; animals were tested by Daniel Fox. Both contributions are gratefully acknowledged. Address requests for reprints to Isaac Behar, USAARL, P.O. Box 577, Fort Rucker, Alabama 36360 .
However, even in sophisticated monkeys, avoidance of the negative stimulus is absent unless the animal has actually responded to it (Behar, 1962; Leary, 1956). The negative stimulus which has not been responded to, when paired with a neutral stimulus, is preferred at levels well above chance expectation. The negative stimulus which has been responded to is avoided, but to a decreasing degree, as the number of intervening error-free trials increases (Behar, 1962).

This waning in avoidance of the negative stimulus with continued training has also been seen by Deutsch \& Biederman (1965) in a paradigm in which the negative stimuli of a relatively more trained (MT) and less trained (LT) discrimination were paired for choice. In that study, 10 of 12 rats at one level of training and 7 of 12 rats at another chose the MT negative stimulus. Since the 
Table 1

Schematization of Experimental Design

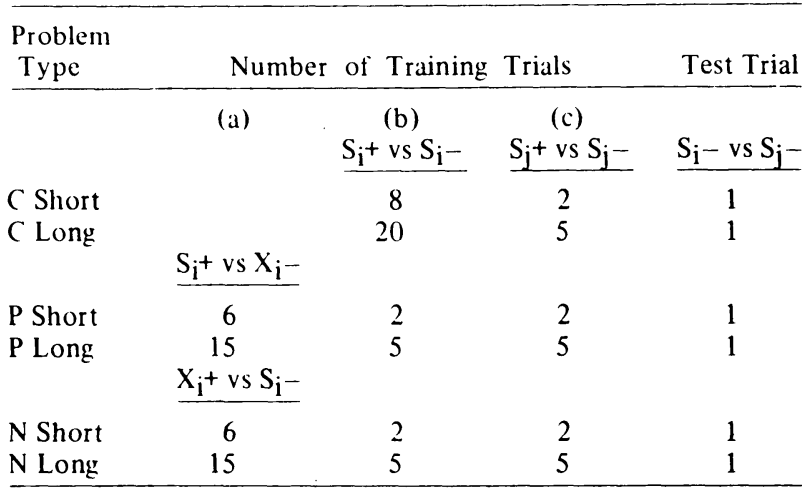

Note-The stimuli designated $X_{i}$ were replaced after the number of trials listed in Column $a$. The trials in Column $a$, if any, preceded those in Columns $b$ and $c$. The trials in Columns $b$ and $c$ were randomly intermixed. The trials in Columns $a$ and $b$ combined constituted the MIT discrimination, Column $c$, the LT discrimination.

rats at the time of choice were still relatively naive, it is unlikely that either negative stimulus was very aversive. Thus, the interpretation that the more frequent choice of the MT negative stimulus was due to its waning aversiveness is confounded by the differential familiarity of the MT and LT negative stimuli. Even if the explanation of the Deutsch and Biederman effect is not this simple, it would still be of interest to determine whether the waning aversiveness of the negative stimulus with continued discrimination experience depends upon its association with $\mathrm{S}+$ or only upon the decreased relative frequency with which it is responded to.

The purposes of the present experiment were twofold: to determine (a) whether the Deutsch and Biederman effect would be obtained with discrimination learning-set sophisticated monkeys, and (b) whether the relative preferences for the negative stimulus of the MT pair could be manipulated by differentially overtraining with only the negative or positive stimulus of the MT pair. In the paradigm employed, when overtraining was with only the positive stimulus, the negative stimulus of the "MT pair" was associated with the more frequently rewarded stimulus, but it and the negative stimulus of the LT pair were equally familiar (i.e., the number of trials on which each was presented was equal). When only the negative stimulus was overtrained, it was more familiar than the negative stimulus of the LT pair; it also had more opportunity for its aversiveness to wane independent of its association with the rewarded stimulus. If overtraining only the positive stimulus leads to a higher choice of the MT negative stimulus than does overtraining only the negative stimulus, then the Deutsch and Biederman effect may be attributed not to the greater familiarity of the MT negative stimulus, but to its association with the more frequently reinforced St. If the opposite result occurs, then this effect is probably due to the greater familiarity of the MT negative stimulus, or, at least, is independent of its association with the MT positive stimulus.

\section{METHOD}

Subjects

Six adult Catarrhine monkeys, three rhesus (Macaca mulatta) and three sooty mangabeys (Cerococebus atys) were used. All were very test sophisticated on problems involving variations of the learning-set paradigm. Their proficiency in solving simultaneous two-choice discriminations is indicated by a practice series of 4210 -trial problems given just prior to the present study. Performance on Trials 2-10 ranged from 97.6\% to $100 \%$ correct responses.

\section{Apparatus}

The Emory version of the WGTA (Riopelle, 1954) was modified so that the raising and lowering of the opaque screen, which separates $\mathrm{S}$ from the test tray, was controlled by a footswitch-actuated, motor-driven lead screw and nut. Timing switches, suitably located, permitted the measurement of response latency. Stimuli consisted of 90 objects which differed from each other in color, shape, and size. No object was used in more than one problem. A continuous white-noise signal effectively masked potentially distracting sounds from outside the test room.

\section{Procedure}

In order to compare the differential effects of positive and negative overtraining, three kinds of problems were used: $C$, or control, problems closely followed the Deutsch and Biederman procedure and provided overtraining of both the positive and negative stimulus; $\mathrm{P}$, or positive, problems provided overtraining of only the positive stimulus; and $\mathrm{N}$, or negative, problems provided overtraining of only the negative stimulus. In addition, two levels of overtraining (10 and 25 discrimination trials) were compared for each problem type.

- The exact procedure for each problem type is schematically presented in Table 1 and is described here:

C Problems. Ss were given a randomly intermixed series of trials on two discrimination problems, e.g., $S_{i}+$ vs $S_{i}-$ and $S_{j}+v s$ $\mathrm{S}_{\mathbf{j}}-$. In the short problems, the MT discrimination was presented on 8 trials while the LT discrimination was presented on 2 trials. In the long problems, the respective numbers of trials were 20 and 5. Following the training trials, a single test trial was presented, which consisted of a pairing of the negative stimuli of the MT and LT discriminations, e.g., $\mathrm{S}_{\mathbf{i}}-$ vs $\mathrm{S}_{\mathrm{j}}$-.

P Problems. As for the control problems, short and long $\mathbf{P}$ problems contained a total of 10 and 25 trials, respectively. For the short problems, the 10 trials consisted first of 6 trials, in which the positive stimulus was paired with a negative stimulus which was then discarded. The positive stimulus was retained and paired with a new negative stimulus. This stimulus pair was presented for two trials randomly intermixed with the LT stimulus pair which also was presented for two trials. In the long $P$ problems, the overtrained positive stimulus was first presented for 15 trials paired with a negative stimulus which was then discarded. The positive stimulus was then paired with a new negative stimulus and presented for 5 trials intermixed with the LT stimulus pair, which also was presented for 5 trials. Following the training trials, the negative stimuli of each discrimination were paired and presented for a single test trial.

$\mathrm{N}$ Problems. The procedure for the $\mathrm{N}$ problems was exactly the same as that for the P problems, except that following 6 or 15 trials, for the short and long problems, respectively, the positive stimuli were discarded, while the negative stimuli were retained and paired with new positive stimuli for the remaining trials. Test trials were the same as those of $C$ and $P$ problems.

Each animal received one problem daily for 18 days. Order of 
presentation of the six problem types was randomized among the six animals with the use of three different randomized Latin squares. Thus. each problem type was assigned to a different animal on a given day. The same stimulus pairs (or triplets) wire used for all problem types on a given day. On any trial. with the opaque screen lowered between the animal and the test tray. the foodwell to be covered by the positive stimulus was baited with a raisin or kernel of corn. Both stimuli were then placed over the foodwells. and the one-way screen was lowered. Next, the opaque screen was raised permitting response. The noncorrection method was used throughout. On the test trials, response to neither stimulus was rewarded.

\section{RESULTS AND DISCUSSION}

The percent of choices, on the test trials, of the MT negative stimulus was independent of problem length: for short and long problems, respectively, it was: C problems. 55.6 and 50.0: P problems, 77.8 and 66.7 : $\mathrm{N}$ problems, 38.9 and 38.9 . The absence of a significant effect of problem length is not unexpected, since, in highly sophisticated monkeys. any training beyond the first trial may be considered overtraining. Species differences were neither expected nor found. Thus, in order to increase the precision of further statistical analyses, results for the short and long problems of each problem type and for the two species were combined.

For the problems in which the positive and negative stimuli were both overtrained, i.e., the $C$ problems, the overall percent choice of the MT negative stimuli was 52.8. Thus, for test-sophisticated monkeys, there is no consistent preference for the MT or LT negative stimuli. This failure to replicate the Deutsch and Biederman finding may be due to the obvious differences of species and test sophistication, but even for the Ss used, a positive finding would be expected since, in another context, evidence for the waning aversiveness of the negative stimulus was found in test-sophisticated monkeys (Behar, 1962). It is possible that the absolute or relative numbers of trials of the MT and LT discriminations were inappropriate since the effect is sensitive to the level of training at which the choice is given (Biederman, 1967; Deutsch \& Biederman, 1965). The present study also differed in the ratio of trials with the MT and LT discrimination: 4 to 1 in the present study instead of 3 to 1 . A final difference is that in the original study only a single test was given, while in the present study 18 such tests were given to each animal under the various conditions. There was, however, no significant trend over blocks of test trials.

Table 2

Distribution of Discrimination Errors

\begin{tabular}{lcccccc}
\hline & \multicolumn{2}{c}{$\mathrm{X}_{\mathrm{i}-}$} & \multicolumn{2}{c}{$\mathrm{S}_{\mathrm{i}-}$} & \multicolumn{2}{c}{$\mathrm{S}_{\mathrm{j}-}$} \\
\hline P Problems & $24^{*}$ & $(.67)$ & 4 & $(.11)$ & 25 & $(.69)$ \\
N Problems & & & $40 \div$ & $(1.11)$ & 25 & $(.69)$ \\
C Problems & & & 22 & $(.61)$ & 25 & $(.69)$ \\
\hline
\end{tabular}

*22/24 on Trials 1 and 2 .

$+21 / 40$ on Trials 1 and $2 ; 14 / 40$ on trials following stimulus substitution.
In the $P$ and $N$ problems. the trials of the LT discrimination were given in the final block of training trials (i.e.. after stimulus substitution occurred) instcad of having been intermixed randomly throughout the training trials. To that extent. the procedure for these problems differs from that of the Deutsch and Biederman study as well as for that of the $C$ problems. Because of this procedural difference. no statistical comparisons were made between the $C$ problems and the $\mathrm{P}$ and $\mathrm{N}$ problems. The differences between the $\mathrm{P}$ and $\mathrm{N}$ problems. however. may be directly compared.

When the pusitive stimulus was overtrained. the overall mean percent ihoices of the MIT negative stimulus was 72.2. In contrast. when the negative stimulus was overtrained. the corresponding percentage was 38.9. The difference in the means for the $P$ and $N$ problems is highly significant $(\mathrm{F}=15.0$. $\mathrm{df}=1.5$. $\mathrm{p}<.01$ ). Thus, the choice of the MT negative stimulus appears to depend not upon its greater familiarity. but upon its association with the more frequently rewarded stimulus.

However, an alternative interpretation derives from the analysis of errors actually committed during the training trials (see Table 2). Since most errors occurred on Discrimination Trials 1 and 2. for the P problems very few responses (a total of 4 . or an average of 0.11 per problem) were made to the "MT" negative stimuli following their introduction. Thus, the actual choice given the animals on the test trials of the P problems was between negative stimuli that had been responded to in a ratio of about 1 to 6 . It is not surprising that they chose the "MT" negative stimulus. For the $\mathrm{N}$ problems, the MT negative stimuli had been responded to 1.6 times as frequently as had the LT negative stimuli. Hence. the relative preference for the latter again is consistent with an explanation based upon a strong tendency for discrimination-sophisticated monkeys to avoid stimuli, associated with nonreward, to which they have responded. Since the Deutsch and Biederman rats had made, prior to the test trial, more nonrewarded responses to the MT than to the LT negative stimulus, their preference for the MT negative stimulus must be based upon other mechanisms.

\section{REFERENCES}

Behar, I. Learned avoidance of nonreward. Psychological Reports, $1961,9,43-52$.

Behar, I. Evaluation of the significance of positive and negative cue in discrimination learning. Journal of Comparative \& Physiological Psychology, 1962, 55, 502-504.

Biederman, G. B. The overlearning reversal effect: A function of the non-monotonicity of the negativity of $\mathrm{S}-$ during discrimination training. Psychonomic Science, 1967, 7, 385-386.

Deutsch, J. A., \& Biederman, G. B. The monotonicity of the negative stimulus during learning. Psychonomic Science, 1965. 3, 301-392.

Leary, $R$. W. The rewarded, the unrewarded, the chosen, and the unchosen. Psychological Reports, 1956, 2, 91-97.

Riopelle, A. J. Facilities of the Emory University Primate Behavior Laboratory. Journal of Psychology, 1954, 38, 331-338.

(Received for publication November 19. 1973.) 